\title{
ÍNDICE DE CONGESTÃO PORTAL E A OCORRÊNCIA DE TROMBOSE PORTAL PÓS-DAPE
}

Fabio Gonçalves ferreira, emuardo Wei Kin Chin, Maria de fatima Santos, Darcy lisbão Moreira de Carvalho, Armando De Capua Junior* Trabalho realizado pelo Grupo de Fígado e Hipertensão Portal do Departamento de Cirurgia da Faculdade de Ciências Médicas da Santa Casa de São Paulo.

* Correspondência:

Av. Comendador Adibo

Ares, 944, São Paulo, SP,

Cep: 05613-001, Telefone:

(11) $\quad 3226-7270$

(11) $7836-7031$.

lauga@terra.com.br

\section{RESUMO}

OBjetivo. Comparar os dados obtidos pela ultra-sonografia com doppler no pré-operatório de esquistossomóticos submetidos à desconexão ázigo-portal com esplenectomia (DAPE), calculando o índice de congestão portal, e sua correlação com a trombose portal no pós-operatório.

Mítooos. Foram estudados 65 pacientes submetidos à DAPE por hipertensão portal esquistossomótica com antecedente de hemorragia digestiva, divididos em dois grupos: Grupo A (28 pacientes que não desenvolveram trombose portal pósoperatória) e Grupo B (37 pacientes com trombose portal no pós-operatório). Analisaram-se através de ultra-sonografia com doppler no pré-operatório os seguintes parâmetros da veia porta: diâmetro, área, velocidade média de fluxo do sangue, fluxo de sangue, e estabeleceu-se 0 índice de congestão portal.

Resultados, $O$ diâmetro, área e 0 fluxo da veia porta foram maiores no grupo $B$ (média de $1,52 \mathrm{~cm} ; 1,77 \mathrm{~cm}^{2} \mathrm{e}$ $2533,12 \mathrm{~m} / \mathrm{min}$ ) em relação ao grupo A (média de $1,33 \mathrm{~cm} ; 1,44 \mathrm{~cm}^{2}$ e 1609,03 $\mathrm{m} / \mathrm{min}$ ) com $p=0,03 ; 0,03$ e 0,04 respectivamente. 0 índice de congestão portal não foi estatisticamente significativo na comparaçãa dos dois grupos $(p=0,07)$.

Conclusia, 0 índice de congestão portal obtido no pré-operatório através da ultra-sonografia com doppler não se mostrou preditivo de trombose portal no pós-operatório dos doentes estudados.

UnIteRMo S: Hipertensão portal. Esquistossomose. Ultra-sonografia. D oppler. Trombose. Veia porta.

\section{INTRODUÇÃO}

A esquistossomose mansônicaé uma doença endêmica no Brasil, que aco mete 8 a 12 milhões de pesso as e expõe 30 milhões ao risco de infecção, sendo que $3 \%$ a $10 \%$ dos infectados desenvolvem a forma hepato esplênica-1.4.

Esta forma crônica da parasitose está relacionada a uma taxa de mortalidade de $12 \%$, chegando a $52 \%$ em algumas séries, nos indivíduos que apresentam hemorragia digestiva alta por varizes esôfago-gástricas ${ }^{5-8}$.

A maneira de intervir na história natural da doença é através do tratamento clínico da helmintíase na forma intestinal e do tratamento cirúrgico para prevenir a recidiva hemorrágica após o primeiro episódio de sangramento nos doentes que desenvolveram hipertensão portal.

A desconexão ázigo-portal com esplenectomia (DAPE) tem proporcionado bons resultados clínicos no tratamento da hemorragia digestiva. ${ }^{5-8}$ Entretanto, este procedimento cirúrgico tem sido acompanhado da ocorrência de trombose pós-operatória do sistema portal $(13,3 \%$ a $53,2 \%)$ que na maioria das vezes tem manifestações brandas, porém é cada vez mais diagnosticada com a utilização da ultra-sonografia com doppler (US-doppler) (,9. $^{8}$.

A alteração hemo dinâmica do sistema portal com a eliminação do fluxo sangüíneo esplênico pela esplenectomia, e a estase condicionada pela desconexão do sistema ázigo-portal podem contribuir para 0 desenvolvimento da trombose do sistema portal no pós-operatório. Acresce-se ainda 0 aumento dos elementos figurados do sangue relacionados à esplenectomia.
A ultra-sonografia com doppler - método simples, de fácil execução, não-invasivo e com ausência de efeitos deletérios ao paciente ${ }^{10,11}$ - torno u-se exame complementar pré e pós-operatório importante nos pacientes submetidos à DAPE, com grande sensibilidade para 0 diagnóstico da trombose portal (TP)9,12.

0 presente estudo visa, através de análise retrospectiva dos pacientes com hipertensão portal esquistossomótica submetidos à desconexão ázigo-portal com esplenectomia, determinar se o índice de congestão portal obtido no pré-operatório teve relação com 0 desenvolvimento de trombose portal no pós-operatório recente.

\section{MÉTODOS}

Realizamos um estudo retrospectivo através de revisão dos prontuários dos pacientes submetidos à desconexão ázigo-portal com esplenectomia no Departamento de Cirurgia da Faculdade de Ciências Médicas da Santa Casa de São Paulo, no perío do de janeiro de 1996 a agosto de 2002.

0 estudo foiaprovado pelo C omitê de Ética em Pesquisaem Seres Humanos da Irmandade da Santa Casa de Misericórdia de São Paulo.

Selecionamos 65 do entes com diagnóstico de esquistossomose mansônica, com hipertensão portal, portadores de varizes gastroesofagianas e antecedente de hemorragia digestiva alta.

0 diagnóstico da doença em sua forma hepatoesplênica baseou-se em dados clínico-epidemiológicos, de exame físico, exame ultrasonográfico hepático, exame proto-parasitológico de fezes (pelo método de Kato-Katz) e, quando necessário, biópsia retal ou mesmo hepática. 
FerReIRA FG et AL.

Todos os doentes foram submetidos a ultra-sonografia com doppler a nível ambulatorial no pré-operatório, bem como avaliação da função hepática, sendo excluídos cinco doentes que apresentaram trombose portal ou hepatopatia de componente misto, pois estes doentes não são submetidos à desconexão ázigo-portal com esplenectomia em nosso serviço.

$0 \mathrm{~s}$ do entes foram operados segundo técnica descrita anteriormente ${ }^{13} \mathrm{e}$ foram reavaliados seqüencialmente através do estudo por ultra-som doppler, além de todos os demais cuidados necessários para o seu acompanhamento clínico cirúrgico.

Realizamos ultra-sonografia com doppler de rotina em todos os doentes no pós-operatório $10^{\circ}$ dia e no $1^{\circ}, 3 \circ, 60$ e $12^{\circ}$ mês após 0 procedimento cirúrgico.

Foi considerada trombose portal no pós-operatório quando era visto trombo no interior da veia porta sem fluxo (trombose total) ou com fluxo diminuído (trombose parcial) ou ainda a transformação caverno mato sa da veia porta indicando a oco rrência prévia de trombose ${ }^{14}$.

0 estudo ultra-so nográfico com doppler foi realizado no Serviço de Diagnóstico por Imagem da Santa C asa de São Paulo, utilizando-se um aparelho de ultra-sonografia bidimensional de tempo real, acoplado ao doppler de onda pulsátil da marca Toshiba®, modelo Sonolayer SSH 140 A, Japão, com transdutor de $3,75 \mathrm{MHz}$.

O s parâmetros ultra-sonográficos analisados no pré-operatório foram: diâmetro da veia porta (DVP); área elíptica da veia porta $\left(\mathrm{cm}^{2}\right)$ calculada pela fórmula ${ }^{15}: A V P=(D V P)^{2} \times \pi / 4$; velocidade do fluxo de sangue na veia porta $(\mathrm{cm} / \mathrm{s})$; fluxo de sangue na veia porta $(\mathrm{ml} / \mathrm{min})$, obtido conforme a fórmula: $F=$ velocidade do fluxo $\times A V P \times 60^{10}, \mathrm{e}$ finalmente 0 índice de congestão portal $(\mathrm{cm} / \mathrm{s})$ pela fórmula proposta por Moriyasu: ICP = AVP / velocidade do fluxo ${ }^{16}$.

$\mathrm{N}$ o pós-operatório, os doentes foram divididos em dois grupos conforme a ocorrência ou não de trombose portal: grupo $A$, composto de 28 doentes sem trombose da veia porta no pós-operatório, e grupo B, formado por 37 doentes com trombose no pós-operatório, diagnosticada através da ultra-sonografia com doppler. No grupo $\mathrm{A}$, a médiade idade foi $38,9 \pm 11,6$ anos com $17(60,71 \%)$ doentes do sexo masculino. lá no grupo $B$ a média de idade foi $39,4 \pm 12,4$ anos com $28(75,68 \%)$ de doentes do sexo masculino (Tabela 1$)$.

As médias e o desvio padrão dos resultados obtidos foram submetidos a análise estatística, utilizando o teste AN 0 VA na comparação entre os grupos, considerando-se $p \leq 0,05$ como nível de rejeição da hipótese de nulidade.

\section{Resultados}

0 diagnóstico de trombose portal foi estabelecido no momento da realização da ultra-sonografia com doppler, em média com $31 \pm 11$ dias de pós-operatório e mediana de 28 dias, havendo predominância de trombose parcial da veia porta em 22 doentes contra 10 que tiveram trombose total (Tabela 2).

A média dos valores do diâmetro, da área e do fluxo de sangue da veia porta foram maiores no grupo $B$ (com trombose) em relação ao grupo $\mathrm{A}$ (sem trombose) com resultado estatisticamente significativo, sendo $p=0,03 ; 0,03$ e 0,04 respectivamente.

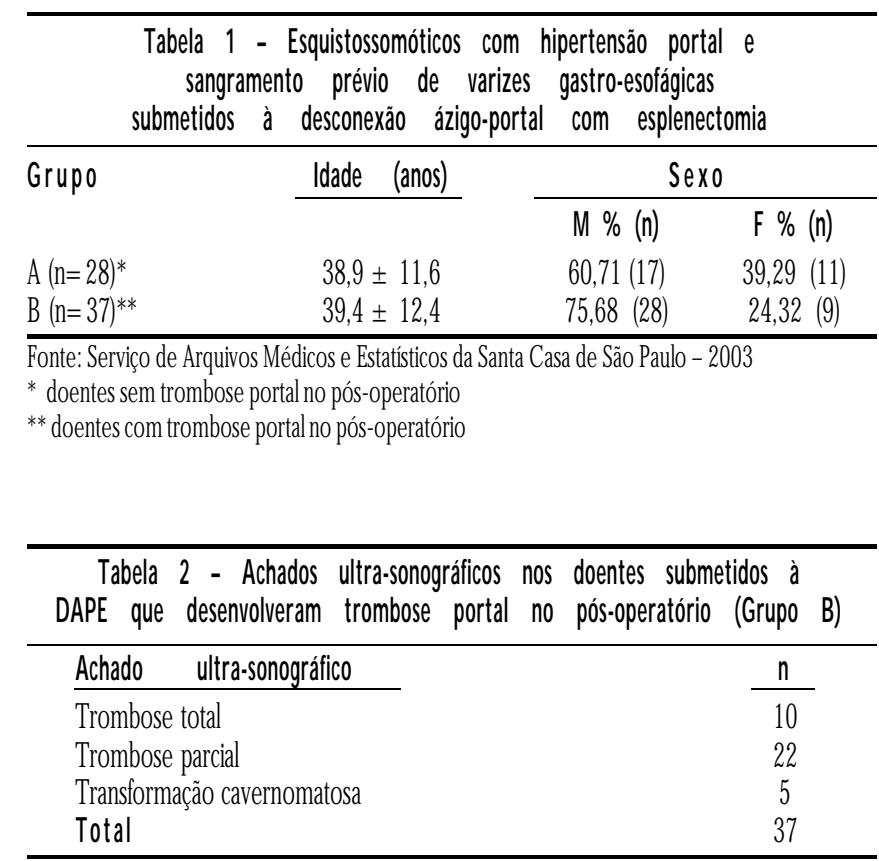

Fonte: Serviço de Arquivos Médicos e Estatísticos da Santa C asa de São Paulo - 2003

Tabela 3 - Médias e desvio padrão dos parâmetros ultra-sonográficos pré-operatórios de 65 esquistossomóticos submetidos à DAPE

\begin{tabular}{|c|c|c|c|c|}
\hline Parâmetro & pré-operatório & Grupo $A^{\S}$ & Grupo $B^{\delta}$ & $p$ \\
\hline \multicolumn{2}{|c|}{ Diâmetro da veia porta (cm) } & $1,33 \pm 0,24$ & $1,52 \pm 0,40$ & 0,03 \\
\hline \multicolumn{2}{|c|}{ Área da veia porta $\left(\mathrm{cm}^{2}\right)$} & $1,44 \pm 0,53$ & $1,96 \pm 1,08$ & 0,03 \\
\hline \multicolumn{2}{|c|}{$\begin{array}{l}\text { Velocidade média de fluxo na } \\
\text { vela porta }(\mathrm{cm} / \mathrm{s})\end{array}$} & $19,21 \pm 4,94$ & $20,05 \pm 6,59$ & $0,09 *$ \\
\hline \multicolumn{2}{|c|}{ Fluxo da veia porta $(\mathrm{m} / / \mathrm{min})$} & $1609,03 \pm 616,75$ & $2533,13 \pm 2239,19$ & 0,04 \\
\hline \multicolumn{2}{|c|}{$\begin{array}{l}\text { Índice de congestão } \\
\text { portal }(\mathrm{cm} / \mathrm{s})\end{array}$} & $0,08 \pm 0,05$ & $0,10 \pm 0,05$ & $0,07 *$ \\
\hline
\end{tabular}

* não significativo

s sem trombose portal no pós-operatório

${ }^{\delta}$ com trombose portal no pós-operatório

Q uando comparamos a média da velocidade do fluxo de sangue na veia porta e a média do índice de congestão portal nos do is grupos 0 resultado não foi estatisticamente significativo, $\operatorname{com} p=0,09 \mathrm{e}$ 0,07 , respectivamente (Tabela 3).

\section{Discussão}

Ao avaliar-se as alterações hemodinâmicas que ocorrem no sistema portal em pacientes esquistossomóticos, o achado mais comum é a elevação da resistência intra-hepática ao fluxo sangüíneo, com redução da fração proveniente da veia porta no fluxo sangüíneo total, havendo ainda um componente de hiperfluxo portal diretamente relacionado à esplenomegalia17,18.

A desconexão ázigo-portal com esplenectomia tem como vantagens corrigir o hiperesplenismo, ser de fácil exeqüibilidade, ter 
menor índice de morbidade e não desenvolver encefalopatia, $6,13,19,20$. Como pontos contrários à sua realização, observou-se maior índice de recidiva hemorrágica e trombose da veia porta ${ }^{21}$.

A fisiopatologia da trombose portal após a desconexão ázigo-portal com esplenectomiatem sido discutida. U madas causas possíveisestaria relacionada à realização da esplenectomia pela redução no fluxo portal causada pela ligadura da veia esplênica. Também deve ser considerada a lesão endotelial causada pela secção e ligadura da veia esplênica?.

A desconexão ázigo-portal com esplenectomia determina a redução do calibre da veia porta e a diminuição do índice portal hepático, caracterizando uma redução do componente de hiperfluxo portal22,23.

A ultra-sonografia com doppler tornou-se o método de escolha para a avaliação do sistema portal por não ser invasiva, possuir baixo custo, ser rápida e reprodutível e possuir grande acurácia em estabelecer o diagnóstico de hipertensão portal. Além disso, é capaz de demonstrar a perviedade da veia porta, a direção do seu fluxo, prover dados anatômicos e fisiológicos (velocidade de fluxo) do sistema venoso portal normal e patológico ${ }^{12,17}$.

0 índice de congestão portal foi introduzido por Moriyasu em 1986, que demonstrou a correlação com a pressão portal em hepatopatias crônicas ${ }^{16}$. Este índice leva em consideração o fluxo de sangue na veia porta e o seu diâmetro, alterações freqüentes na hipertensão portal. Vários fatores podem alterar o índice de congestão: pressão na veia porta, resistência intra-hepática, fluxo de sangue portal e circulação colateral portossistêmica.

Em nosso estudo, o índice de congestão portal foi maior no grupo $B$ quando comparado ao do grupo $A$, apesar de não haver significância estatística. Contudo, ambos os grupos apresentaram valores superiores ao dos indivíduos normais $(0,07 \mathrm{~cm} / \mathrm{s})^{11,16}$.

0 estudo com US-doppler no pós-operatório é realizado em

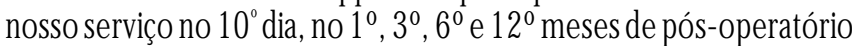
e, ocasionalmente, quando presentes sintomas sugestivos de trombose da veia porta. Desse modo, consideramos a transformação cavernomatosa como indicativo de um fenômeno trombótico em recanalização, e esses doentes mantidos como portadores de trombose portal e seus dados ultrassonográficos pré-operatórios analisados no grupo $B$.

Todos os parâmetros analisados através da ultra-sono grafia com doppler demonstraram média de valores superiores para 0 grupo que desenvolveu trombose no pós-operatório $(B)$ em relação ao grupo sem trombose (A), porém somente o diâmetro, aárea o o fluxo de sangue da veia porta tiveram significância estatística.

$\mathrm{N}$ ão há dúvida de que a esquistossomose mansônica em sua forma hepatoesplênica promove alteração hemodinâmica no sistema portal. N ele existe uma acomodação estrutural, a fim de manter-se 0 fluxo de sangue constante. Isto pôde ser observado em nosso estudo pela presença de diâmetro e área de veia porta acima dos valores de normalidade nos pacientes submetidos à DAPE.

Após o procedimento cirúrgico, houve redução no diâmetro e no fluxo de sangue da veia porta ${ }^{12,24}$. N ova adaptação hemodinâmicadeve ocorrer de forma que se mantenha perfusão hepática satisfatória. A trombose portal pode corresponder à inadequada adaptação circulatória causada pela abrupta redução do fluxo portal após a retirada do baço.
Os achados de diâmetro, área e fluxo de sangue na veia porta superiores nos doentes que desenvolvem trombose da veia porta no pós-operatório parecem indicar que estes indivíduos apresentam maior resistência hepática ao fluxo de sangue, e que, após o procedimento cirúrgico, a adaptação à nova condição hemodinâmica não é satisfatória, promovendo fenômenos trombóticos, às vezes passando até despercebidos tanto pelo doente quanto pelo médico, já que a trombose parcial na maioria das vezes é assintomática.

Assim, 0 índice de congestão portal, maior nos doentes com trombose portal pós-operatória, apesar de não estatisticamente significativo, tem valor na avaliação pré-operatória dos esquistossomóticos. A simples observação dos dados que compõem este índice (diâmetro, área e fluxo portais) pode prever já no pré-operatório se devemosnos preocupar com atrombose portal, podendo indicar o uso rotineiro de anticoagulantes nesses doentes, apesar dos riscos de sangramento pelas varizes. Estudosna coagulação destes doentes estão sendo realizados em nosso serviço, podendo interferir na história natural da trombose portal no pós-operatório da DAPE.

\section{CONCLUSÃO}

O s resultados obtidos nas condições de execução deste trabalho nos permitiram concluir que 0 índice de congestão portal obtido no pré-operatório através da ultra-sonografia com doppler não se mostrou preditivo de trombose portal no pós-operatório dos doentes estudados.

Conflito de interesse: não há.

\section{SUMmarY}

\section{Portal CONGESTION AND thrombosIS after EDS}

BACKGROUND. The study compared the preoperative portal vein congestion index estimated by Doppler ultrasound and the postoperative portal vein thrombosis of patients submitted to esophagogastric devascularization and splenectomy(EDS).

Meth ODS. 65 patientswith portal hypertension due to schistosomiasis and previo us gastrointestinal bleeding submitted to EDSweredivided into two groups: GROUPA (28 patients without postoperative portal vein thrombosis) and GROUP B (37 patients with postoperative portal vein thrombosis). The following parameters of preoperative Doppler ultrasound ofthe portal vein were analyzed: diameter, area, mean blood flow velocity and blood flow, whereupon the congestion index wascalculated.

RESU LTS. The diameter, area and blood flow of the portal vein were greater in group $B$ (mean of $1.52 \mathrm{~cm} ; 1.77 \mathrm{~cm}^{2}$ and $2533.12 \mathrm{ml} / \mathrm{min}$ ) than in group $A$ (mean of $1.33 \mathrm{~cm} ; 1.44 \mathrm{~cm}^{2}$ and $1609.03 \mathrm{ml} / \mathrm{min}$ ) with $p=$ $0.03 ; 0.03$ and 0.04 respectively. Difference ofthe congestion index was not statistically significant between the two groups $(p=0.07)$.

CONCLU SION S. The portal vein congestion index at the preoperative of EDS estimated by Doppler ultrasound was not predictive of portal vein thrombosis in the postoperative of patients with portal hypertension dueto schisto somiasis. [Rev Assoc Med Bras 2005; 51(4): 233-6]

KEY wO RDS: Portal hypertension. Schistosomiasis. U Itrasound. Doppler. Thrombosis. Portal vein. 
FERREIRA FG ET AL.

\section{REFERÊNCIAS}

1. Prata A. Significado da esquistosso mose para o Brasil. Rev Assoc Med Bras 1975, 21(10):301-2.

2. Prata A Infection with S. mansoni. In: Jordan, P.; Webbe, G. Schistosomiasis: epidemiology, treatment and control. London, W illiam H einemann, 1982. p.105-257.

3. Bina CJ A expansão da esquistossomose mansoni no Brasil: fatores determinantes e sugestões para o seu controle. Rev Med Bahia 1976, 22(2):86-100.

4. Barberato Filho S, Gargioni C, Pinto PLS, Chiodelli RG, Vellosa SAG, Silva $\mathrm{RM}$, et al. Synthesis and evaluation of new oxaminiquine derivatives. Int J Pharmaceutics 2002, 233: 35-41.

5. Kelner S, Ferreira PR, D antas A, Lima Filho JFC, So uza AP, C arreiro Junior $J C P$, et al. Ligadura de varizes esôfago-gástricas na hipertensão porta esquistossomótica: avaliação de 25 anos. Rev Col Bras Cir 1982, $9(1): 140-6$.

6. Assef JC, De Capua Junior A, Szutan LA Treatment of recurrent hemorrhagic esophageal varices in schistosomotic patients after surgery. Rev Assoc Med Bras 2003, 49(4):406-12.

7. Szutan LA. Resultados imediatos e tardios da esplenectomia e desvascularização esofago gástrica no tratamento da hemorragia digestiva alta em esquistossomóticos [dissertação]. São Paulo: Faculdade de Ciências Médicas, Santa C asa de São Paulo; 1993.

8. Pugliese V. Desconexão ázigo-portal e esplenectomia associadas à escleroterapia endoscópica no tratamento das varizes do esôfago na esquistossomose hepato-esplênica: avaliação de parâmetros clínicos, laboratoriais e hemodinâmicos portais [dissertação]. São Paulo: Faculdade de Medicina, U niversidade de São Paulo; 1996.

9. Santos MF. Trombose da veia porta em doentes portadores da esquistossomose mansônica: forma hepatoesplênica - submetidos a desconexão ázigo -portal e esplenectomia: análise da incidência, fatores de risco, evolução clínica e ultra-sonográfica. [dissertação]. São Paulo: Faculdade de Ciências Médicas, Santa Casa de São Paulo; 2002.

10. Brown HS, Halliwell M, Q amar M, Read AE, Evans JM, W ells PNT. Measurement of normal portal venous blood flow by $D$ oppler ultrasound. Gut 1989, 30(5):503-9.

11. EidtJF, H award t, Cook JM, Kahn MB, Troillett R. C urrent status of duplex D oppler ultrasound in the examination of the abdominal vasculature. Am J Surg 1990, 160(6):604-9.

12. Carvalho D LM. Fluxo venoso portal e função hepática pós esplenectomia e desconexão ázigo-portal [dissertação]. São Paulo: Faculdade de C iências Médicas, Santa Casa de São Paulo; 2002.

13. De Capua Jr A. Desconexões ázigo-portais. In: Aspectos técnicos na cirurgia do aparelho digestivo. São Paulo. Robe; 1991. p.185-8.
14. De G aetano AM, Lafortune M, Patriquin H, D e Franco A, Aubin B, Paradis K. Cavernous transformation of the portal vein: patterns of intrahepatic and splanchnic collateral circulation detected with doppler sonography. AJR Am J Roentgenol 1995,115(3):389-93.

15. Vezozzo DCP. Avaliação hepática e hemodinâmica portal com Doppler duplex na esquistossomose mansônica [disssertação]. São Paulo: Faculdade de Medicina, U niversidade de São Paulo; 1992.

16. Moriyasu $F, N$ ishida $O$, Ban $N, N$ akamura $T$, Sakai $M$, Miyake $T$, et al. "Congestion Index" of the portal vein. AJR Am J Roentgenol 1986,146(7): 735-9.

17. Cerri GG. Contribuição da ultra-sonografia no diagnóstico da forma hepatoesplênica da esquistossomose mansônica [dissertação]. São Paulo: Faculdade de Medicina, U niversidade de São Paulo; 1984.

18. Sacerdoti D, Merkel C, Bolognesi M, Amodio P, Angeli P, G atta A. H epatic arterial resistance in cirrhosis with and without portal vein thrombosis: relationships with portal hemodynamics. Gastroenterology 1995, 108(4):1152-8.

19. D e C apuaJr A, Szutan LA. D esconexão ázigo-portal e esplenectomia mais escleroterapia no tratamento da hipertensão portal. C lin Bras Cir 1995, 2(2):231-42.

20. Assef JC. Recidiva hemorrágica após operações não descompressivas para tratamento de hemorragia digestiva alta em esquistossomóticos [dissertação]. São Paulo: Faculdade de Ciências Médicas, Santa Casa de São Paulo; 1992.

21. Chandler JG. The history of the surgical treatment of portal hypertension. Arch Surg 1993, 128(9):925-40.

22. Lacerda CM, Ramos H, Raia S, Kelner S. Fisiopatologia da hipertensão portal esquistossomótica e efeitos da esplenectomia com ligadura de varizes de esôfago. Acta Cir Bras 1993, 8(3):113-7.

23. Widman A, O liveira IRS, Speranzini MB, Cerri GG, Saad WA, GamaRodrigues J. Hipertensão portal por esquistossomose mansônica hepatoesplênica: efeito da desconexão ázigo-portal com esplenectomia no diâmetro e na velocidade média de fluxo do sistema portal (estudo ultra-sonográfico com D oppler). Arq Gastroenterol 2001, 38(1):19-23.

24. Alves Júnior A, Alves MD N T, G onçalves KRA, C ruz JF, M elo VA, Machado MCC. Hipertensão portal esquistossomótica: avaliação do fluxo sangüíneo portal antes e após tratamento cirúrgico. Rev Col Bras Cir 2001, 28(5):330-335.

Artigo recebido: 05/05/04 Aceito para publicação: 18/10/04 